\title{
ATTRACTION BETWEEN BROWNIAN PARTICLES OF IDENTICAL CHARGE IN COLLOID CRYSTALS
}

\author{
P.A. KRALCHEVSKY, N.D. DENKOV, I.B. IVANOV and A.D. NIKOLOV \\ Laboratory of Thermodynamics and Physico-Chemical Hydrodynamics, University of Sofia, Faculty of Chemistry, \\ Sofia 1126, Bulgaria
}

Received 17 August 1989; in final form 3 January 1990

\begin{abstract}
A new source of attraction between similarly charged colloidal particles in ordered suspensions is considered. The attractive force is due to the correlation between the fluctuating displacements of the colloidal particles from their equilibrium positions in the colloid crystal lattice. The effective charge of a particle, the eigenfrequency and the energy of attraction are calculated on the basis of a dynamic model of the counterion atmosphere.
\end{abstract}

\section{Introduction}

It has been established experimentally by many authors [1-8] that spherical particles of submicron size in monodisperse latex suspensions form crystallike ordered structures. These structures, called "colloid crystals" [9], differ from the usual (molecular) crystals by the size of the particles and by the magnitude of the interparticle distances. In particular, the interparticle distances are too large for the attractive van der Waals forces to be important. That is why some authors [10-13] attribute the observed disorder-order phase transition only to the screened electrostatic repulsion between the charged latex particles. A phase coexistence and phase boundary between ordered and disordered domains, which are not due to sedimentation, were detected in several experiments [6-8]. Sogami and Ise [14] pointed out that a phase coexistence is possiblc when there is a net attractive interaction between the colloidal particles. They attempted to reveal the nature of this attraction by ascribing it to Coulombic interaction between the macroions through the intermediary of counterions. A different possibility for attraction, connected with charge fluctuations on the surface of an ordered phase, was discussed by Imai and Yoshino [15-17].

We propose now a new effect which can give rise to attraction between the charged particles in colloid crystals. The basic idea is that a fluctuating displacement of a particle from the centre of the counterion atmosphere produces a fluctuating dipole. This dipole can interact with a neighboring fluctuating dipole. Also, the displacement of one charged particle can disturb the charge distribution around a neighboring particle and induce an additional dipole moment. We show below that the total dipole-dipole interaction (both between spontaneous and induced dipoles) averaged over time leads to attraction. In this respect this effect resembles the London dispersion forces between molecules.

We suppose that, besides the attractive energy, each colloidal particle in the colloid crystal has another potential energy

$U_{0}(s)=\frac{1}{2} m \omega_{0}^{2} s^{2}$

corresponding to harmonic oscillation. Here $m$ and $s$ are the mass of the particle and the magnitude of its displacement from the centre of the potential well; $\omega_{0}$ has the dimensions of frequency. In section 4 below, the potential energy $U_{0}(s)$ is attributed to the electrostatic attraction between the effective centres of the positive and negative charges: the charged colloidal particle and the centre of its counterion atmosphere. Such an interaction gives rise to a quasielastic force $f=\mathrm{d} U_{0} / \mathrm{d} s$ tending to restore the equi- 
librium position of the particle at the centre of the well.

\section{Fluctuations in a colloid crystal lattice}

Each colloidal particle in a colloid crystal is encircled by the "atmosphere" of the counterions dissociated from the particle surface. Because of the fluctuating motion of the particle around the centre of the potential well the effective centres of positive and negative charges do not coincide. Thus, a fluctuating dipole appears. The moment $p$ of such a dipole for small fluctuations is proportional to the displacement $s$ of the particle from the well centre:

$p=q s$,

where the proportionality constant $q$ has the dimensions of electrical charge. A dynamic model of the countcrion atmospherc allowing calculation of $q$ is presented in sections 4 and 5 .

Let $s^{(1)}$ and $s^{(2)}$ be the instantaneous displacements of two particles in the colloid crystal and let $\boldsymbol{R}$ be the vector connecting the two resulting dipoles,

$p^{(1)}=q s^{(1)}, \quad p^{(2)}=q s^{(2)}$.

Then the dipole-dipole interaction energy will be [18]

$U_{\mathrm{p}}=m \omega_{\mathrm{p}}^{2}\left[s^{(1)} \cdot s^{(2)}-\left(3 / R^{2}\right)\left(s^{(1)} \cdot R\right)\left(s^{(2)} \cdot R\right)\right]$,

where

$\omega_{\mathrm{p}}^{2}=q^{2} / m \in R^{3}$

with $\epsilon$ the dielectric permittivity of the liquid medium. Our purpose now is to derive equations allowing calculation of $U_{\mathrm{p}}$. For the sake of simplicity we first consider a one-dimensional crystal and we then generalize the results to three dimensions.

Let $R$ be the nearest-neighbor separation for a onedimensional "colloid crystal". The fluctuating particle experiences four forces: two stemming from the energies $U_{0}$ and $U_{\mathrm{p}}$, the viscous friction force and the random Brownian force. Then, in view of eqs. (1) and (4) the equation of motion of particle $n$ reads

$x_{n}+\beta x_{n}+\omega_{0}^{2} x_{n}-2 \omega_{\mathrm{p}}^{2}\left(x_{n-1}+x_{n+1}\right)=A_{n}(t)$,

where $x_{n}$ is the position of particle $n$ with its own lat- tice point chosen as origin; $\beta$ is a coefficient characterizing the force of friction and $A_{n}(t)$ is the acceleration due to the Brownian force. Only the interaction between nearest neighbors is accounted for in eq. (6). Let us define the Fourier transforms $\tilde{x}$ and $\tilde{A}$ of $x_{n}$ and $A_{n}$ by the equations

$$
\begin{aligned}
& x_{n}(t)=\int_{-\infty}^{\infty} \mathrm{d} \omega \int_{-\pi}^{\pi} \mathrm{d} k \tilde{x}(\omega, k) \exp [\mathrm{i}(n k-\omega t)], \\
& A_{n}(t)=\int_{-\infty}^{\infty} \mathrm{d} \omega \int_{-\pi}^{\pi} \mathrm{d} k \tilde{A}(\omega, k) \exp [\mathrm{i}(n k-\omega t)] .
\end{aligned}
$$

Here $k$ is a dimensionless wave number. Substituting in eq. (6) we obtain

$$
\left(-\omega^{2}-\mathrm{i} \beta \omega+\omega_{k}^{2}\right) \tilde{x}(\omega, k)=\tilde{A}(\omega, k)
$$

with

$$
\omega_{k}^{2}=\omega_{0}^{2}-4 \omega_{\mathrm{p}}^{2} \cos k \text {. }
$$

Using the approach of Landau and Lifshitz $[19,20]$, we can write the correlation of the Brownian accelerations as

$\overline{A_{n}(t) A_{l}\left(t^{\prime}\right)}=\frac{2 \beta k_{\mathrm{B}} T}{m} \delta\left(t-t^{\prime}\right) \delta_{n t}$.

The presence of the term $\delta\left(t-t^{\prime}\right) \delta_{n l}$ means that the Brownian accelerations of different particles at different times are independent [19]. The coefficient $2 \beta k_{\mathrm{B}} T / m$ is determined from the condition $\overline{\bar{x}_{n}^{2}}=k_{\mathrm{B}} T /$ $m$ ( $k_{\mathrm{B}}$ is the Boltzmann constant, $T$ the temperature), see ref. [21]. After a Fourier transformation eq. (11) takes the form

$\overline{\widetilde{A}(\omega, k) \tilde{A}\left(\omega^{\prime}, k^{\prime}\right)}=\frac{\beta k_{\mathrm{B}} T}{2 m \pi^{2}} \delta\left(\omega+\omega^{\prime}\right) \delta\left(k+k^{\prime}\right)$.

The displacement correlation is calculated by means of cq. (7), along with (9) and (12) (see ref. [20] for details),

$\overline{x_{n}(t)} \overline{x_{l}(t)}=\frac{\beta k_{\mathrm{B}} T}{2 m \pi^{2}} \int_{-\infty}^{\infty} \mathrm{d} \omega \int_{-\pi}^{\pi} \frac{\cos [(n-l) k] \mathrm{d} k}{\left(\omega_{k}^{2}-\omega^{2}\right)^{2}+\beta^{2} \omega^{2}}$. 
The averaged energy of attraction for two nearest neighbors is calculated from eq. (4) written for the particles $n$ and $n-1$,

$$
\bar{U}_{\mathrm{p}}=-2 m \omega_{\mathrm{p}}^{2} \overline{x_{n}(t) x_{n-1}(t)} \text {. }
$$

Solving the integral in (13) for $l=n-1$, we thus obtain

$$
\begin{aligned}
& \bar{U}_{\mathrm{p}} / k_{\mathrm{B}} T=\frac{1}{2}\left[1-I_{1}(\xi)\right], \quad I_{1}(\xi)=1 / \sqrt{1-16 \xi^{2}}, \\
& \xi-\omega_{\mathrm{p}}^{2} / \omega_{0}^{2}
\end{aligned}
$$

Similarly, one can determine

$\overline{x^{2}}=\overline{x_{n}(t) x_{n}(t)}=\frac{k_{\mathrm{B}} T}{m \omega_{0}^{2}} I_{1}(\xi)$.

Note that $I_{1}(\xi)>1$ and hence $\bar{U}_{\mathrm{p}}<0$, i.e. $U_{\mathrm{p}}$ corresponds to attraction.

This method for calculating of $\overline{x^{2}}$ and $\bar{U}_{\mathrm{p}}$ can be directly generalized for a three-dimensional simple cubic lattice. The results in this case read

$\overline{s^{2}}=3 \overline{x^{2}}=\frac{3 k_{\mathrm{B}} T}{m \omega_{0}^{2}} I(\xi)$,

$\bar{U}_{\mathrm{p}} / k_{\mathrm{B}} T=\frac{1}{2}[1-I(\xi)]$,

where

$I(\xi)=\frac{1}{\pi^{3}} \int_{0}^{\pi} \int_{0}^{\pi} \int_{0}^{\pi} \mathrm{d} k_{1} \mathrm{~d} k_{2} \mathrm{~d} k_{3}\left|\Phi\left(k_{1}, k_{2}, k_{3}, \xi\right)\right|^{-1}$,

$\Phi\left(k_{1}, k_{2}, k_{3}, \xi\right)=1+2 \xi\left(\cos k_{2}+\cos k_{3}-2 \cos k_{1}\right)$.

As earlier $\bar{U}_{\mathrm{p}}$ is the averaged energy of dipole-dipole interaction between two nearest neighbors in the colloid crystal lattice. For $\xi \rightarrow 0$ one has $I(\xi) \approx 1$ and eqs. (16) and (17) both lead to the known formula $\overline{x^{2}}=k_{\mathrm{B}} T / m \omega_{0}^{2}$ valid for a Brownian oscillator [22]. Besides, for small $\xi$ by expanding the function $\Phi$ in terms of $\xi$, and by carrying out the integration in eq. (19), taking also into account eqs. (5) and (18), one can derive

$\bar{U}_{\mathrm{n}} \approx-6 k_{\mathrm{B}} T q^{4} / \epsilon^{2} m^{2} \omega_{0}^{4} R^{6}$.

Hence, similarly to the energy of van der Waals interactivin, $\bar{U}_{\mathrm{p}}$ is proportional to $R^{-6}$ (for $\omega_{\mathrm{p}}^{2} \ll \omega_{0}^{2}$ ) and corresponds to attraction.
A closer inspection of the integral $I(\xi)$ in eq. (19) shows that

$$
\begin{aligned}
& I(\xi)=\left(8 \xi \pi^{2}\right)^{-1} \int_{0}^{\pi} \mathrm{d} k_{3} \alpha\left(k_{3}\right) K\left(\alpha\left(k_{3}\right)\right), \\
& \quad \text { for } 0 \leqslant \xi \leqslant \frac{1}{8}, \\
& I(\xi)=\infty, \quad \text { for } \xi>\frac{1}{8} .
\end{aligned}
$$

Here $K(\alpha)$ is the total elliptic integral of Legendre of the first kind and

$$
\alpha\left(k_{3}\right)=\xi \sqrt{32}\left[\left(1-2 \xi \cos k_{3}\right)^{2}-4 \xi^{2}\right]^{-1 / 2} .
$$

The fact that the integral $I(\xi)$ is divergent at $\xi>\frac{1}{8}$ in conjunction with eq. (17) indicates infinite motion of the Brownian particle, i.e. "melting" of the colloid crystal. The value $\xi=\frac{1}{8}$ corresponds to a limiting value of the mean-squared displacement:

$s_{l}=\left(\frac{3 k_{\mathrm{B}} T}{m \omega_{0}^{2}} I\left(\frac{1}{8}\right)\right)^{1 / 2} \approx 1.26\left(\frac{3 k_{\mathrm{B}} T}{m \omega_{0}^{2}}\right)^{1 / 2}$.

Correspondingly, in a stable colloid crystal the averaged energy of interaction between two neigboring dipoles, $\bar{U}_{\mathrm{p}}$, (cf. eq. (18)) cannot exceed the limiting value $\left|\bar{U}_{\mathrm{p}}\left(\xi=\frac{1}{8}\right)\right|=0.29 k_{\mathrm{B}} T$ - see fig. 1 .

Although limited, this attractive energy is comparable to the thermal energy $k_{\mathrm{B}} T$. It is difficult to say to what extent this energy will play a role in the behavior of colloid crystals. Our aim was to propose in the present paper a new source of interparticle attraction in such systems and to develop a theory for its calculation. According to our theory the energy $\bar{U}_{\mathrm{p}}$ is a strong function of the parameters $q$ and $\omega_{0}$

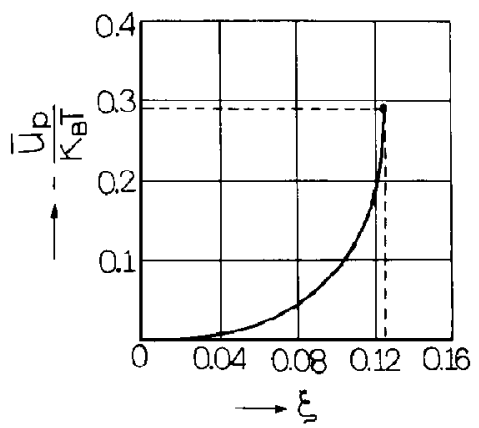

Fig. 1. The averaged dipole-dipole interaction energy, $\bar{U}_{\mathrm{p}}$, between two neighboring particles versus $\xi=\omega_{\mathrm{p}}^{2} / \omega_{0}^{2}$ in a colloid crystal of simple cubic lattice. 
(see e.g. eq. (21)), which in turn depend on the specific system under consideration.

\section{Cell model of a colloid crystal}

A latex crystal can be considered as an assembly of unit cells each containing one charged colloid particle - fig. $2 a$. The particle is encircled by the atmosphere of the dissociated counterions the whole cell being electroneutral.

To simplify our treatment we approximate a unit polyhedral cell with a sphere of the same volume. The radius of this sphere is denoted by $R_{2}$ in fig. 2 . In the same figure the sphere of radius $R_{1}$ contains the colloid particle itself as well as the nearest counterions, which move together with the particle: we call $R_{1}$ the hydrodynamic radius of the particle. Let us denote by $Q_{\text {eff }}$ the magnitude of the electric charge of the particle, i.e. the net charge, contained in the sphere of radius $R_{1}$. Duc to the fluctuations the particle centre at a given moment will be at a distance $s$ from the cell centre - fig. $2 b$. The particle motion will perturb the ionic atmosphere so that its centre of charge will not coincide with the cell centre. If the distance between the charge centres is $h$, the instantaneous dipole moment is $p=Q_{\text {efr }} h$. We assume $h$ and $s$ are proportional for small displacement, i.e.

$h=\mathrm{D} h /\left.\mathrm{d} s\right|_{0} s+\mathfrak{C}\left(s^{2}\right)$.

Then the expression for the dipole moment can be written as $p \approx q s, \quad q \approx Q_{\text {eff }}(\mathrm{d} h / \mathrm{d} s)_{s=0}$,

cf. eq. (2). The attraction between the effective centres of the positive and negative charge gives rise to a quasi-elastic force which tends to return the particle to the centre of the cell; the harmonic potential energy can be expressed by eq. (1). The physical origin of the quasi-elastic energy $U_{0}$ is discussed in more detail below.

In section 2 we showed that the mean-square displacement of a Brownian particle from its equilibrium position in a colloid crystal (with simple cubic lattice) can be calculated from eq. (17). A comparison between eq. (18) and fig. 1 reveals that $I(\xi) \geqslant I(0)=1$. Thus eq. (17) leads to an estimate for $\omega_{0}$ :

$\omega_{0}^{2} \geqslant 3 k_{\mathrm{R}} T / m \bar{s}^{2}$.

$\overline{s^{2}}$ can be calculated from the experimental data of Ito et al. [23] for ordered latex suspensions. By using an ultramicroscope and image data analyzer there authors determined the quantity

$G=2\left(\frac{1}{3} s^{2}\right)^{1 / 2} / R$.

For an ordered suspension with $8 \%$ volume fraction of Latex N400 they found $R=730 \mathrm{~nm}$, and $G=0.021$ [23]. From their data one can calculate $m=3.52 \times 10^{-14} \mathrm{~g}$, so that from eqs. (26) and (27) one finds $\omega_{0} \geqslant 0.8 \times 10^{6} \mathrm{~s}^{-1}$ at room temperature. The relaxation time of the counterion atmosphere is of the order of $10^{-3} \mathrm{~s}[24]$. This large difference in time

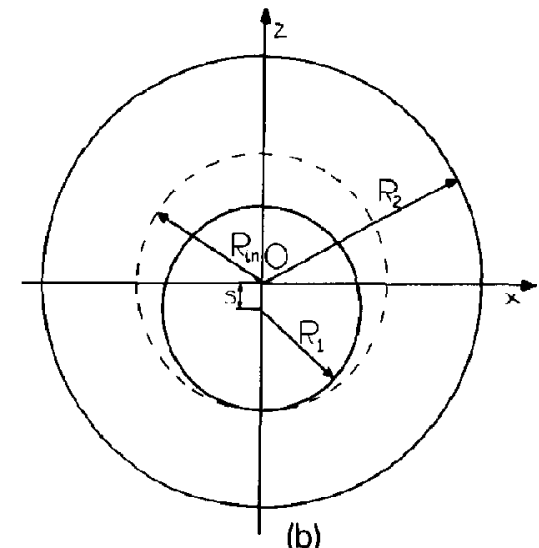

(b)

Fig. 2. Cell model of an ordcred latex suspension: a time-averaged picture (a), and an instantaneous displacement of a particle from the cell centre (b). 
thus obtained are shown in table 1 for two different concentrations of Latex $N 400$. The frequency $\omega_{0}$ is of the order of $10^{6} \mathrm{~s}^{-1}$ and $Q_{\mathrm{eff}} / e$ is smaller than $2.3 \times 10^{3}$. These values confirm the above estimate based on eqs. (26) and (38). The "width" of the counterion atmosphere $\chi^{-1}$ is comparable to the particle radius $R_{1}$. Also, $\xi<\frac{1}{8}$ and $\overline{s^{2}}<s_{R}^{2}$ as it should be for a stable colloid crystal. $\bar{U}_{\mathrm{p}}$ is of the order of $0.1 k_{\mathrm{B}} T$ for the more concentrated suspension.

\section{Model of the counterion atmosphere}

One can assume that a colloidal particle, moving rapidly in the potential well, acts like a stirrer: it makes the ion concentration homogeneous in the close vicinity of the particle. Let $\rho_{\text {in }}$ be the absolute value of this homogeneous charge density - see fig. 3. Further, because of the long relaxation time of the ionic atmosphere we assume that the charge distribution in the remaining part of the cell maintains its spherical symmetry. Because of the attraction between the particle and the dissociated counterions, the concentration of the latter should decrease with $r$ (see fig. 3). Then,

$\rho_{\text {in }} \geqslant \bar{\rho}$,

wherc

$\bar{\rho}=Q_{\mathrm{eff}} / \frac{4}{3} \pi\left(R_{2}^{3}-R_{1}^{3}\right)$

is the absolute value of the mean charge density in the cell.

Consider the force

$f=\int_{r_{\text {cell }}} \frac{r_{12}}{\epsilon r_{12}^{3}} Q_{\text {cff }} \rho\left(r_{2}\right) \mathrm{d} r_{2}$

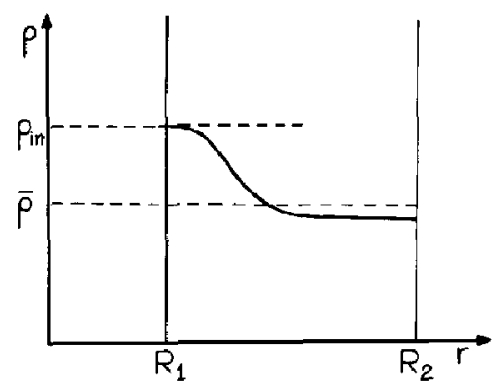

Fig. 3. Sketch of the charge distribution of the counterions around a colloid particle of hydrodynamic radius $R_{1}$. of interaction of a colloid particle ( of charge $Q_{\text {efr }}$ situated at $\boldsymbol{r}_{1}$ ) with its counterion atmosphere of charge density $\rho$; in eq. (30) $\boldsymbol{r}_{12}=r_{2}-\boldsymbol{r}_{1}, \boldsymbol{r}_{12}=\left|\boldsymbol{r}_{12}\right|$. The integration in eq. (30) is carried out over the volume between the spheres of radii $R_{1}$ and $R_{2}-$ fig. $2 \mathrm{~b}$. If the $z$ axis is oriented as shown in ig. $2 \mathrm{~b}$, then the magnitude of $f$ can be expressed as

$f=\int_{V_{\text {cell }}} \frac{z_{12}}{\epsilon r_{12}^{3}} Q_{\text {eff }} \rho\left(r_{2}\right) \mathrm{d} r_{2}$.

In the framework of the present model $\rho$ has spherical symmetry with respect to the centre of the cell - see fig. 3. Hence the integration over the volume outside the sphere of radius $R_{\text {in }}=R_{1}+s$ (fig. $2 \mathrm{~b}$ ) does not contribute to the force $f$. Besides, $\rho=\rho_{\text {in }}=$ const. inside this sphere. Then the integral in eq. (31) can be easily calculated:

$f=(4 \pi / 3 \epsilon) Q_{\text {eff }} p_{\text {in }} s$.

This is the quasi-elastic force acting on the particle. Since eq. (1) yields

$f=\mathrm{d} U_{0} / \mathrm{d} s=m \omega_{0}^{2} s$

one obtains the expression for the frequency $\omega_{0}$ :

$\omega_{0}^{2}=(4 \pi / 3 \epsilon m) Q_{\mathrm{eff}} \rho_{\text {in }}$.

If the charge of the particle is negative (which is the case with latexes), the centre of the negative charge is at $z^{(-)}=-s$ (fig. 2b). The distance $h=z^{(+)}-z^{(-)}$between the effective centres of the positive and negative charges is

$h=\left(\int_{V_{\text {cll }}} z \rho(r) \mathrm{d} r\right) / Q_{\text {eff }}+s$.

By carrying out the integration one obtains

$h=\left(4 \pi R_{1}^{3} \rho_{\text {in }} / 3 Q_{\text {eff }}+1\right) s+\mathcal{C}\left(s^{2}\right)$.

A combination of eqs. (25) and (35) yields

$q=Q_{\text {eff }}+\frac{4}{3} \pi R_{1}^{3} \rho_{\text {in }}$.

The elimination of $\rho_{\text {in }}$ between eqs. (33) and (36) leads to a relationship between $q$ and $\omega_{0}$ :

$q=Q_{\mathrm{eff}}+\left(m \epsilon / Q_{\mathrm{eff}}\right) R_{1}^{3} \omega_{0}^{2}$.

From eqs. (28), (29) and (33) one derives

$Q_{\mathrm{cff}} \leqslant\left[\epsilon m \omega_{0}^{2}\left(R_{2}^{3}-R_{1}^{3}\right)\right]^{1 / 2}$, 
which provides an upper limit for the effective charge of a colloid particle. Since $I(\zeta) \leqslant I\left(\frac{1}{8}\right)=1.58$ (cf. eq. (22)), by using eqs. (17), (27), (38) and the data for the latex studied in ref. [23] one calculates $Q_{\mathrm{eff}} /$ $e \leqslant 2.3 \times 10^{3}$ ( $e$ is the magnitude of the electron charge). This number is about $1 \%$ of the total number of surface ionizable groups per particle given in ref. [23].

\section{Determination of $\rho_{\text {in }}$}

We still need one more equation for determining $\rho_{\text {in }}$. To obtain such an equation we assume that

$\rho(r)=\rho_{\text {in }}, \quad$ for $R_{1} \leqslant r<R_{1}+\bar{s}$,

$\rho(r)=\rho_{\text {in }} \exp \left(-\frac{z e \psi(r)}{k_{\mathrm{B}} T}\right), \quad$ for $R_{1}+\bar{s}<r \leqslant R_{2}$,

where $\bar{s}=\left(\overline{s^{2}}\right)^{1 / 2}$ and $\psi(r)$ is the electrostatic potential, satisfying the Poisson equation

$\Delta \psi=-(4 \pi / \epsilon) p(r)$

with boundary conditions

$\psi\left(R_{1}+\bar{s}\right)=0, \quad \mathrm{~d} \psi / \mathrm{d} r\left(r=R_{2}\right)=0$.

Eq. (40) holds for colloidal particles whose ionic atmosphere contains only one kind of ions of charge $z e$. (Eq. (40) can be generalized for the case when electrolyte has been added to the solution.) The condition for electroneutrality of a cell reads

$Q_{\mathrm{err}}=\left|\int_{R_{1}}^{R_{2}} 4 \pi r^{2} \rho(r) \mathrm{d} r\right|$.

By using the linearised version of eq. (40)

$\rho(r)=\rho_{\text {in }}\left[1-\left(e z / k_{\mathrm{B}} T\right) \psi(r)\right]$,

$$
\text { for } R_{1}+\bar{s} \leqslant r \leqslant R_{2} \text {, }
$$

one can easily solve eq. (41) along with the boundary conditions (42). Then in vicw of cq. (44) one transforms eq. (43) to

$$
\begin{gathered}
Q_{\mathrm{eff}}=\mid \rho_{\mathrm{in}}\left\{\frac{4}{3} \pi\left[\left(R_{1}+\bar{s}\right)^{3}-R_{1}^{3}\right]\right. \\
\left.+4 \pi\left[F\left(R_{2}\right)-F\left(R_{1}+\bar{s}\right)\right]\right\} \mid,
\end{gathered}
$$

where

$$
\begin{aligned}
& F(r)=\left(R_{1} / \chi^{2}\right)[C(\chi)(\chi r-1) \exp (\chi r) \\
& -C(-\chi)(\chi r+1) \exp (-\chi r)], \\
& C(\chi)=\left(1+\frac{\chi R_{2}-1}{\chi R_{2}+1} \exp \left[2 \chi\left(R_{2}-R_{1}\right)\right]\right)^{-1} \\
& \quad \times \exp \left(-\chi R_{1}\right), \\
& \chi^{2}=4 \pi e z^{2} \rho_{\text {in }} / \epsilon k_{\mathrm{B}} T .
\end{aligned}
$$

Eq. (45) yields the sought for connection between $\rho_{\text {in }}$ and $Q_{\text {eff- }}$

\section{Numerical results and discussion}

The parameters $R, R_{1}, R_{2}, m, \epsilon$ and $T$ are usually known from experiment. From the data of Ito et al. [23], one can calculate $\overline{s^{2}}$ by using eq. (27). Then one can determine the four parameters $\omega_{0}, q, Q_{\text {eff }}$ and $\rho_{\text {in }}$ from the four equations (17), (33), (37) and (45). The use of this procedure along with the data of Ito et al. [23] is not entirely self-consistent, for eq. (17) refers to a particle in the bulk of a simple cubic lattice, whereas $\bar{s}^{2}$ was determined in ref. [23] for particles situated at the boundary of a facecentred-cubic lattice with the glass wall of the experimental cell. The parameters $\xi, s_{l}$ and $\chi$ can be calculated by means of eqs. (5), (15), (24) and (48). We calculated the averaged energy of attraction between two nearest neighbors in the colloid crystal, $\bar{U}_{\mathrm{p}}$, from eq. (18). The integral $I(\xi)$ in eq. (22) was calculated numerically. In all cases we used $\epsilon=78.2$ and $T=298 \mathrm{~K}$. The values of the parameters

Table 1

Parameter values for two different concentrations of Latex N400

\begin{tabular}{lcc}
\hline Concentration & $\begin{array}{l}2 \% \text { volume } \\
\text { fraction }\end{array}$ & $\begin{array}{l}8 \% \text { volume } \\
\text { fraction }\end{array}$ \\
\hline$R_{1}(\mathrm{~nm})$ & 200 & 200 \\
$R(\mathrm{~nm})$ & 1070 & 730 \\
$R_{2}(\mathrm{~nm})$ & 591 & 403 \\
$\left(s^{2}\right)^{1 / 2}(\mathrm{~nm})$ & 27 & 14 \\
$s_{\ell}(\mathrm{nm})$ & 34 & 16 \\
$\chi^{-1}(\mathrm{~nm})$ & 205 & 116 \\
$Q_{\mathrm{eff}} / e$ & 532 & 717 \\
$q / e$ & 620 & 994 \\
$\omega_{0}\left(\mathrm{~s}^{-1}\right)$ & $7.0 \times 10^{5}$ & $1.5 \times 10^{6}$ \\
$\xi$ & 0.054 & 0.102 \\
$-\bar{U}_{\mathrm{p}} / k_{\mathrm{B}} T$ & 0.019 & 0.093 \\
\hline
\end{tabular}


N400. The frequency $\omega_{0}$ is of the order of $10^{6} \mathrm{~s}^{-1}$ and $Q_{\text {eff }} / e$ is smaller than $2.3 \times 10^{3}$. These values confirm the above estimate based on eqs. (26) and (38). The "width" of the counterion atmosphere $\chi^{-1}$ is comparable to the particle radius $R_{1}$. Also, $\zeta<\frac{1}{8}$ and $\overline{s^{2}}<s_{Q}^{2}$ as it should be for a stable colloid crystal. $\bar{U}_{\mathbf{p}}$ is of the order of $0.1 k_{\mathrm{B}} T$ for the more concentrated suspension.

Additional theoretical analysis of the importance of $\vec{U}_{\mathrm{p}}$ for ordering in latex suspensions is needed. We intend to investigate the role of the new attractive forces on the phase behavior and other properties of microheterogeneous systems containing charged particles.

\section{Acknowledgement}

This work was supported by the Bulgarian Committee for Science and Education.

\section{References}

[1] W. Luck, M. Klier and H. Wesslaw, Ber. Bunsenges. Physik. Chem. 67 (1963) 75.

[2] P.A. Hiltner and I.M. Krieger, J. Phys. Chem. 73 (1969) 2386.

[3] J.W. Vanderhoff, H.J. Van den Hul, R.J.M, Tausk and J.Th.G. Overbeek, in: Clean surfaces: their preparation, and characterization for interfacial studies, ed. G. Goldfinger (Dekker, New York, 1970).

[4] S. Hachisu, Y. Kobayashi and A. Kose, J. Colloid Interface Sci. 46 (1973) 342.
[5] K. Furusawa and N, Tomutsu, J. Colloid Interface Sci. 93 (1983) 504.

[6] K. Ito, H. Nakamura and N. Ise, J. Chem. Phys. 85 (1986) 6136.

[7] K. Ito, H. Nakamura and N. Ise, J. Chem. Phys. 85 (1986) 6146.

[8] T. Yoshiyama and I. Sogami, Langmuir 3 (1987) 851.

[9] P. Pieranski, Contemp. Phys. 24 (1983) 25.

[10] W. van Megen and I. Snook, Advan. Colloid Interface Sci. 21 (1984) 119.

[11] S. Marčelja, D.J. Mitchell and B.W. Ninham, Chem. Phys. Letters 43 (1976) 353.

[12] T. Ohtsuki, S. Mitaku and K. Okano, Japan. J. Appl. Phys. 17 (1978) 627.

[13] Y. Monovoukas and A. Gast, J. Colloid Interface Sci. 128 (1989) 533 .

[14] I. Sogami and N. Ise, J. Chem. Phys. 81 (1984) 6320.

[15] N. Imai and S. Yoshino, J. Colloid Interface Sci. 105 (1985) 492.

[16] S. Yoshino, in: Ordering and organisation in ionic solutions, eds. N. Ise and I. Sogami (World Scientific, Singapore, 1988) p. 449.

[17] N. Imai, in: Ordering and organisation in ionic solutions, eds. N. Ise and I. Sogami (World Scientific, Singapore, 1988) p. 522.

[18] W. Kauzmann, Quantum chemistry (Academic Press, New York, 1957).

[19] E.M. Lifshitz and L.P. Pitaevsky, Theoretical physics, Vol. 9. Statistical physics, Part 2 (Nauka, Moscow, 1978), in Russian.

[20] L.D. Landau and E.M. Lifshitz, Fluid mechanics (Pergamon Press, Oxford, 1984) p. 528.

[21] R. Becker, Theorie der Warme (Springer, Berlin, 1955).

[22] S. Chandrasekhar, Rev. Mod Phys. 15 (1943) 1.

[23] K. Ito, H. Nakamura and N. Ise, J. Am. Chem. Soc. 110 (1988) 6955 .

[24] G. Schwarz, J. Phys. Chem. 66 ( 1962$) 2636$. 